\title{
Apoptosis induced by replication inhibitors in Chk1- depleted cells is dependent upon the helicase cofactor Cdc45
}

\author{
R Rodriguez ${ }^{1}$, ME Gagou ${ }^{1}$ and M Meuth ${ }^{\star, 1}$
}

Checkpoint kinase 1 (Chk1) responds to disruption of DNA replication to maintain the integrity of stalled forks, promote homologous recombination-mediated repair of replication fork lesions, and control inappropriate firing of replication origins. This response is essential for viability as replication inhibitors trigger apoptosis in S-phase cells depleted of Chk1. Given the complex network of cellular responses controlled by Chk1, our aim was to determine which of these protect cells from apoptosis following replication stress. Work with cell-free systems has shown that RPA-ssDNA complex forms following replication inhibition through the uncoupling of replication and helicase complexes. Here we show that replication protein A (RPA) foci form in cells treated with replication inhibitors and that the number of foci dramatically increases together with hyperphosphorylation of RPA34 in Chk1-depleted cells in advance of the induction of apoptosis. RPA foci, RPA34 hyperphosphorylation, and apoptosis were suppressed by siRNA-mediated knockdown of Cdc45, an essential replication helicase cofactor required for both the initiation and elongation steps of DNA replication. In contrast, loss of p21, a negative effector of origin firing, stimulates both the accumulation of RPA foci and apoptosis. Taken together, these results suggest that the loss of control of replication origin firing following Chk1 depletion triggers the accumulation of the RPA-ssDNA complex and apoptosis when replication is blocked.

Cell Death and Differentiation (2008) 15, 889-898; doi:10.1038/cdd.2008.4; published online 1 February 2008

Cells have a complex response to DNA damage and replication fork stress enabling them to detect of changes in DNA structure and initiate signal transduction pathways that activate cell-cycle checkpoints, recruit DNA repair proteins, and/or trigger apoptosis. ${ }^{1}$ The $\mathrm{PI}(3) \mathrm{K}$-like kinases Ataxia-Telangiectasia Mutated (ATM) and ATM and Rad3 related (ATR) are central to this response. ${ }^{2}$ ATM rapidly reacts to DNA double strand breaks (DSBs) induced by agents such as ionizing radiation. ATR and its downstream phosphorylation target, checkpoint kinase 1 (Chk1), are generally activated in response to UV and agents that stall DNA replication forks. ${ }^{1,3}$ Both ATR and Chk1 are required for the cellular response to disruptions of DNA synthesis and dysfunction of either protein reduces the viability of cells exposed to agents that cause replication fork stress. ${ }^{4-6}$ Recent work provides compelling evidence that this pathway is triggered as a result of the functional uncoupling of polymerase and helicase activities following disruption of DNA synthesis. ${ }^{7}$ Single-stranded DNA (ssDNA) formed by the continued activity of the helicase complex in the absence of replication is coated with replication protein $A$ (RPA) that, in turn, interacts with the ATR-interacting protein (ATRIP) and ATR. $^{8,9}$ The formation of this complex is necessary for efficient phosphorylation and activation of Chk $1,{ }^{8}$ although Chk1 also may be phosphorylated by ATR where ATR-ATRIP complex does not bind to RPA. ${ }^{9}$ Activated Chk1 coordinates many of the cellular responses to replication fork stress. More specifically, it prevents inappropriate firing of late replication origins, abandonment of replication forks, and premature chromosome condensation following disruption of replication. $^{6,10-12}$ Chk1 activation has also been shown to be required for the homologous recombination (HR)-mediated restart of replication forks stalled by replication inhibitors ${ }^{13}$ and to prevent the induction of apoptosis. ${ }^{6,14-16}$

Previous work from our group has focused on how loss of these pathways affects the commitment of cells to apoptosis following the disruption of DNA replication. Many replication inhibitors are normally poor inducers of apoptosis in cultured tumor cells. However, we ${ }^{16}$ and others ${ }^{14,15}$ have shown that siRNA-mediated ablation of Chk1 (but not checkpoint kinase 2) causes cells arrested in S-phase by replication inhibitors to rapidly undergo apoptosis. This death response is p53 independent, but cells that lack both Chk1 and p21 show a more robust death response and reduced cell survival. ${ }^{16}$ Thus, the Chk1 pathway plays a key role in protecting S-phase cells from undergoing apoptosis during replication stress, and p21 mediates this role by preventing entry into S-phase. Given the complex network of responses regulated by Chk1 following replication fork stress, the purpose of this investigation was to determine which of them control the induction of apoptosis. Here we show the effects of disruption of HR or replication initiation on this response.

\footnotetext{
${ }^{1}$ Institute for Cancer Studies, University of Sheffield, School of Medicine and Biomedical Sciences, Sheffield S10 2RX, UK

*Corresponding author: M Meuth, Institute for Cancer Studies, The University of Sheffield, Medical School, Beech Hill Road, Sheffield S10 2RX, UK. Tel: + 44114271 3288; Fax: + 44114271 3515; E-mail: m.meuth@ sheffield.ac.uk Keywords: DNA damage response; apoptosis; S-phase checkpoints; DNA replication

Abbreviations: ATM, Ataxia-Telangiectasia Mutated; ATR, ATM and Rad3 related; Chk1, checkpoint kinase 1; DSB, double strand break; HR, homologous recombination; HU, hydroxyurea; MMR, mismatch repair; RPA, replication protein A; siRNA, specific interfering RNA; ssDNA, single-stranded DNA

Received 18.5.07; revised 21.12.07; accepted 21.12.07; Edited by S Kornbluth; published online 01.2.08
} 


\section{Results}

Cells defective in HR do not become committed to apoptosis following disruption of DNA replication. Given the report that Chk1 is required for HR induced by replication inhibitors, ${ }^{13}$ we first tested the hypothesis that disruption of HR-mediated rescue of replication forks stressed by treatment with replication inhibitors committed cells to apoptosis. siRNA-mediated knockdowns of the known HR proteins XRCC3 and Rad51 were achieved in a strain of the human colorectal carcinoma cell line SW480 carrying the SCneo recombination reporter (SW480/SN3) after a 24-h treatment (Figure 1a). To determine whether these knockdowns affected HR, these cells were then transfected with an expression construct for I-Scel endonuclease that introduces a single DSB in one of the defective neo genes in the reporter. This break is resolved by homology-driven recombination to produce a functional neo gene that allows the formation of neo ${ }^{+}$colonies. $^{17}$ In SW480/SN3 cells depleted of XRCC3 or Rad51, the frequency of neo ${ }^{+}$recombinants was reduced 10- and 40-fold, respectively, relative to that obtained in cells treated with the control siRNA (Figure 1b). Chk1 depletion produced a 2.5 -fold reduction in the level of neo ${ }^{+}$recombinants, consistent with the earlier report. ${ }^{13}$ Thus, siRNA-mediated knockdowns of proteins required for HR suppress the level of recombination in this sensitive assay. To determine the effect of knockdowns of XRCC3 or Rad51 on the induction of apoptosis following excess thymidine treatment, we measured the fraction of SW480/SN3 cells with a subG1 DNA content by flow cytometry following depletion of XRCC3, Rad51, or Chk1. Cells depleted of the two HR proteins showed little or no change in the fraction of cells having a subG1 DNA content following thymidine treatment, whereas those depleted of Chk1 showed a clear increase (Figure 1c). These results are consistent with a previous report from our group ${ }^{18}$ showing that the apoptotic response of hamster cells deficient in XRCC2, XRCC3, or Rad51C to replication inhibitors was not enhanced relative to that obtained with parental cells or cells corrected for the repair defect. Thus, the HR status of the cells does not appear to be a major determinant of apoptosis induction following disruption of DNA replication.

RPA foci accumulate in Chk1-depleted cells as an early response to DNA replication fork stress that precedes the induction of apoptosis. Chk1 activation, which is triggered following formation of RPA-sSDNA regions, controls further accumulation of such intermediates by preventing the inappropriate firing of late replication origins. We first determined whether this RPA-ssDNA complex is formed in thymidine-treated cells by measuring the accumulation of RPA in chromatin and RPA foci. We then determined whether Chk1 depletion caused a further accumulation of the RPA-ssDNA complex after treatment with replication inhibitors as a potential indicator of the inappropriate firing of replication origins. Soluble and chromatin-bound proteins were fractionated from cultures of the human colon cancer cell line HCT116 treated with thymidine for analysis of RPA. The level of RPA34 and RPA70 in the chromatin-bound fraction increased weakly in cells treated with the control siRNA following exposure to thymidine (Figure 2). In Chk1-depleted cells, the increase in the chromatin-bound RPA proteins was more pronounced and the increase in RPA34 was largely in the form of the hyperphosphorylated protein. Despite the increase in RPA34 in the nuclear fraction, there was no change in the overall level of this protein (Supplementary Information; Figure 1).

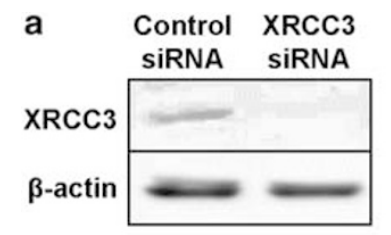

b

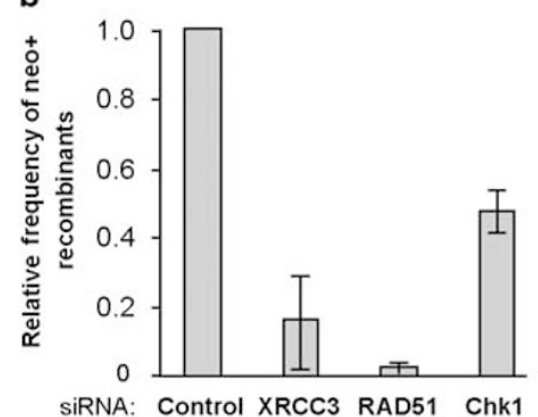

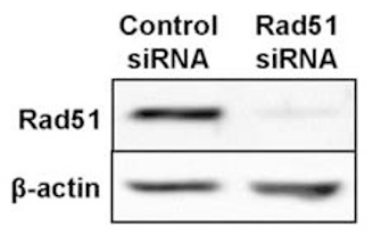

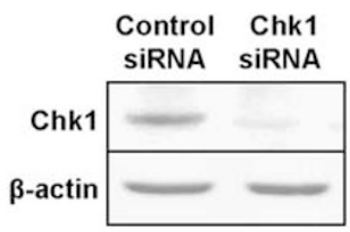

c

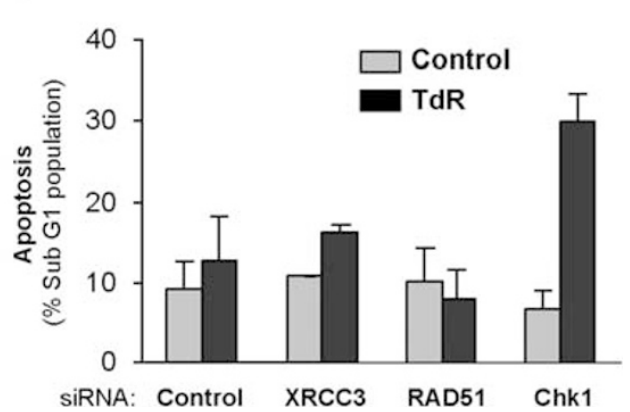

Figure 1 DNA replication stress does not promote apoptosis in HR-deficient cells. (a) Depletion of HR proteins in SW480/SN3 cells treated with the indicated siRNAs for $24 \mathrm{~h}$. $\beta$-Actin levels are presented as a loading control. (b) Frequency of neo ${ }^{+}$recombinants obtained after transfection of an expression construct for endonuclease I-Scel into SW480/SN3 cells treated with the indicated siRNA for $24 \mathrm{~h}$. The frequency of neo ${ }^{+}$recombinants in cells treated with control siRNA and I-Scel expression construct was $0.7-1.3 \times 10^{-3}$, whereas the frequency of neo ${ }^{+}$recombinants in cells not treated with the I-Scel expression construct was lower than $10^{-6}$ in every condition. (c) Percentage of apoptotic subG1 cells in SW480/SN3 cultures exposed to $2 \mathrm{mM}$ thymidine (TdR) following transfection with the indicated siRNAs 


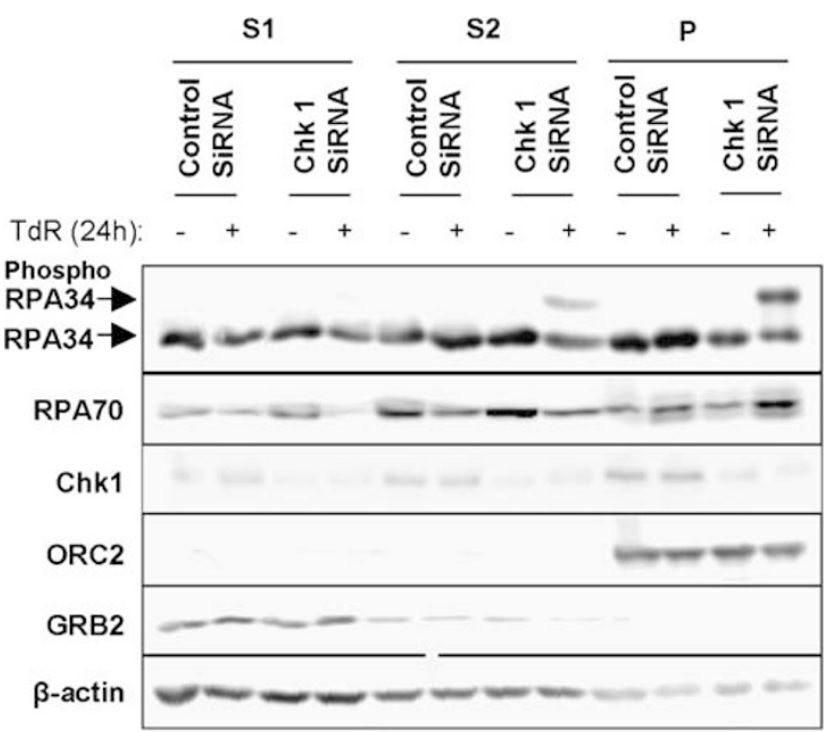

S1 - Cytosolic fraction

S2 - Soluble nuclear fraction

$P$ - Chromatin-bound fraction

Figure 2 RPA accumulates in chromatin in Chk1-depleted cells following replication fork stress. HCT116 cells were transfected with the indicated siRNAs, and treated or not treated with $2 \mathrm{mM}$ thymidine $(\mathrm{TdR})$ for $24 \mathrm{~h}$. Cell fractionation was carried out as indicated in Materials and Methods and the levels of RPA34, RPA70, and Chk1 present in cytosolic (S1), the soluble nuclear (S2), and the chromatinbound $(P)$ fractions were analyzed by western blotting. The hyperphosphorylated form of RPA34 is indicated. ORC2 and GRB2 were used as markers for the chromatin-bound $(\mathrm{P})$ and cytoplasmic $(\mathrm{S} 1)$ proteins, respectively. ${ }^{19} \beta$-Actin levels are presented as loading controls

There was a weak but significant increase in the fraction of HCT116 cells containing low levels of RPA foci $(<10$ per cell) as early as $1 \mathrm{~h}$ after thymidine treatment (Figure $3 \mathrm{a}$ and b). In Chk1-depleted cells, RPA foci were strikingly more numerous and more intense following thymidine treatment. Cells showing the high level of foci ( $>10$ per cell) were evident as early as $1 \mathrm{~h}$ after treatment and continued to accumulate through $24 \mathrm{~h}$, where they represented about $50 \%$ of the population (Figure 3b). RPA foci that formed in Chk1depleted cells treated with thymidine colocalized with PCNA (Figure $3 \mathrm{c}$ ), indicating that they were present at replication forks. While the fraction of cells showing RPA foci increased in Chk1-depleted cells treated with thymidine, the fraction of cells developing Rad51 foci (>10 per cell) decreased (Figure 3d; Supplementary Information; Figure 2), consistent with previous reports. ${ }^{13}$

Importantly, the accumulation of RPA foci in Chk1-depleted cells preceded the induction of apoptosis (Figure 3e). At $24 \mathrm{~h}$ post thymidine treatment, there was only a small increase in the frequency of Annexin $\mathrm{V}+$ cells in cultures treated with the Chk1 siRNA relative to those treated with the control, whereas $\sim 50 \%$ of cells presented $>10$ RPA foci. At $48 \mathrm{~h}$ post thymidine treatment, $60 \%$ of cells were Annexin $\mathrm{V}+$. Similarly, measurements of apoptosis using the TUNEL assay revealed a low level of TUNEL + cells $(1-2 \%)$ in cultures treated with either control or Chk1 siRNAs through a 24-h exposure to thymidine (Supplementary Information;
Figure 3). However, by $48 \mathrm{~h}$, there was an increased accumulation of TUNEL + cells in cultures treated with the Chk1 siRNA relative to cultures treated with the control. Thus, the level of RPA in chromatin and RPA foci significantly increased after thymidine treatment of Chk1-depleted cells in advance of the induction of apoptosis.

We also considered the possibility that the induction of RPA foci might be the result of damage triggered by thymidine in Chk1-depleted cells. Although thymidine is not a DNAdamaging agent and does not induce detectable DSBs, ${ }^{20,21}$ we determined whether DSBs accumulated in Chk1-depleted cells. Untreated, Chk1-depleted, and Chk1-depleted cells treated with thymidine were gently lysed in agarose blocks and DNA was fractionated by pulsed-field gel electrophoresis (Figure 4). No DNA fragments characteristic of damageinduced DSBs were detected in Chk1-depleted cells treated with thymidine in this assay, although smaller fragments resembling those formed during apoptosis were seen in these cells.

Cdc45 depletion suppresses the accumulation of RPA foci in Chk1-depleted cells following DNA replication stress. Although these results were consistent with the model that Chk1 prevents the further accumulation of RPAssDNA complex by controlling the inappropriate firing of replication origins, we further tested this hypothesis by examining the distribution of the replication helicase cofactor Cdc45 in Chk1-depleted cells, and by determining the effect of depletion of this protein on the accumulation of RPA foci. Cdc45 is a gene essential for the initiation of DNA replication in yeast ${ }^{22}$ and DNA unwinding during replication elongation in Xenopus egg extracts. ${ }^{23}$ Loss of either function would be predicted to reduce the accumulation of RPA coated SSDNA and RPA foci in the presence of replication inhibitors. ${ }^{23,24}$ In yeast, Cdc45 is predominantly associated with replication and not $\mathrm{HR}$-mediated repair of replication fork damage. ${ }^{25}$ Previous reports have shown increased loading of Cdc45 onto chromatin following treatment with Chk1 inhibitors in some human cell lines, consistent with an increased initiation of DNA replication. ${ }^{26} \mathrm{~A}$ similar increase in the level of chromatin-bound Cdc45 was detected in Chk1depleted HCT116 cells treated with thymidine, relative to cells treated with the control siRNA (Figure 5a).

Considering the critical role of Cdc45 in DNA synthesis, we next determined the effect of siRNA-mediated depletion of this protein in unstressed cells. Following a 24-h siRNA treatment (Figure $5 b$ ) in the absence of any replication inhibitor, HCT116 cells depleted of Cdc45 showed an increase in the level of Sphase cells and a lesser increase in the level of cells with a G2 DNA content (Figure 5C). However, this was not sufficient to trigger Chk1 phosphorylation (Supplementary Information; Figure 4) or significantly alter the level of cells with a subG1 DNA content (Figure 5c). Thus, transient siRNA-mediated depletion of Cdc45 appears to slow S-G2 progression, but does not significantly affect cell viability or induce damage.

To determine the effect of $\mathrm{Cdc} 45$ depletion on the formation of the RPA-ssDNA complex in Chk1-depleted cells, HCT116 cells treated with Cdc45 and/or Chk1 siRNAs for $24 \mathrm{~h}$ (Figure 5a) were then exposed to thymidine for a further $24 \mathrm{~h}$ before they were assayed for chromatin-bound RPA or 
untreated
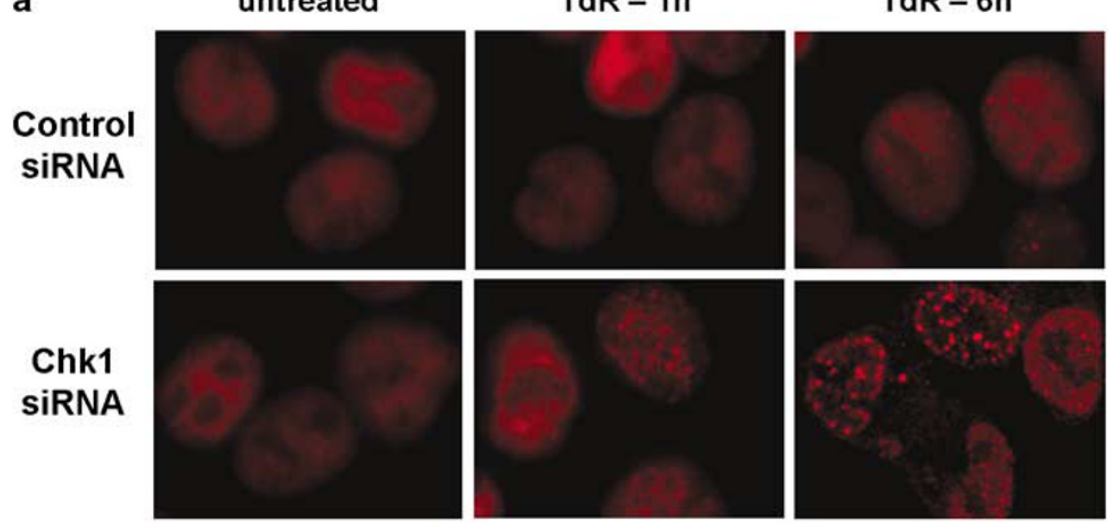

b

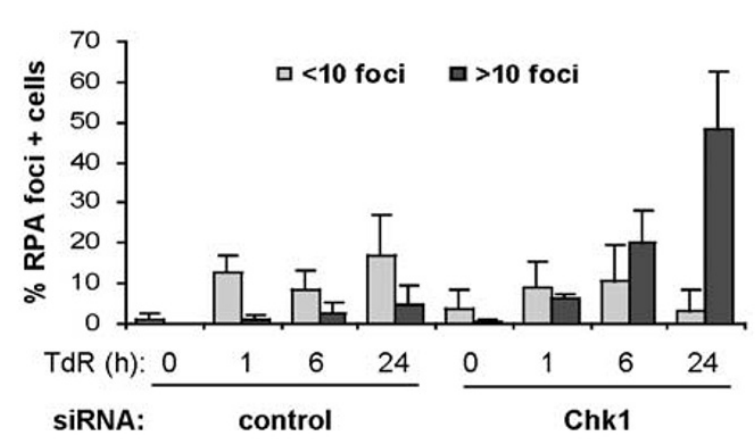

C

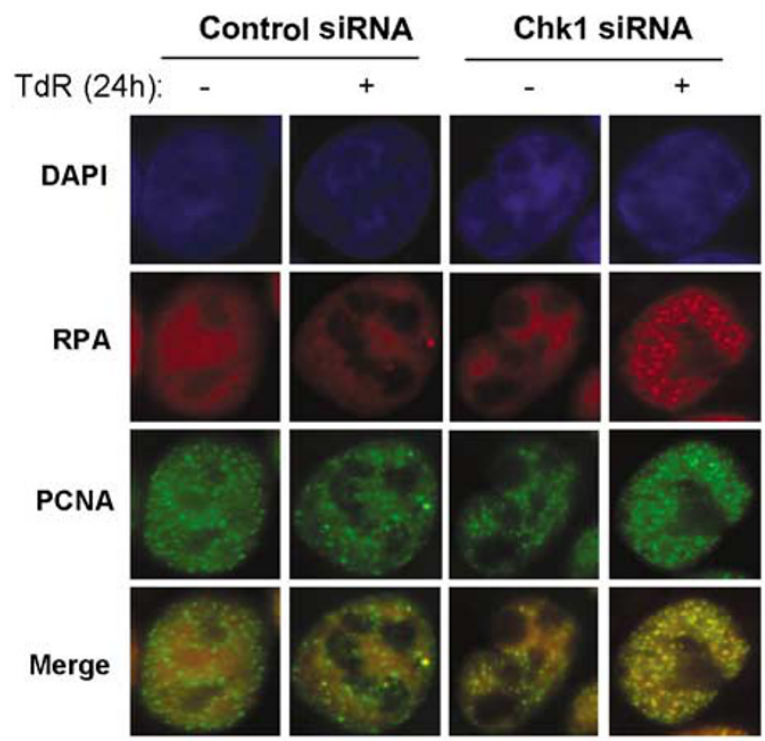

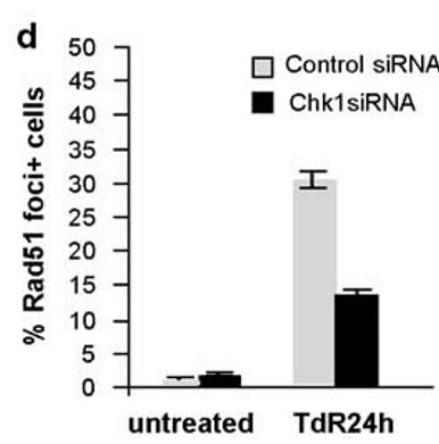

e

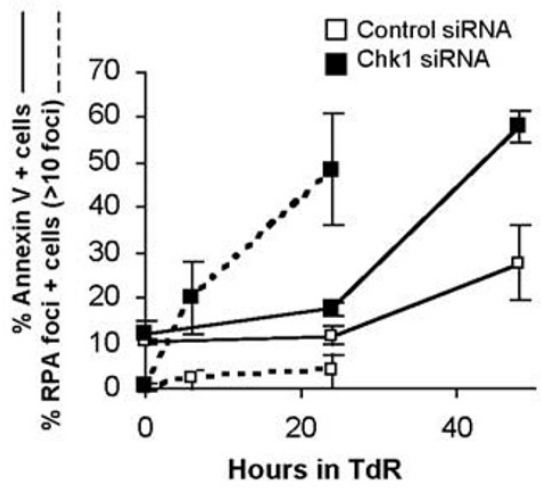

Figure 3 RPA foci accumulate in Chk1-depleted cells after replication fork stress. HCT116 cells were transfected with the indicated siRNAs and treated or not treated with $2 \mathrm{mM}$ thymidine (TdR) for the indicated times and analyzed for (a and $\mathbf{b})$ immunostaining of RPA34. (a) Representative images showing the increased level of RPA foci in Chk1-depleted cells. RPA foci are evident as early as $1 \mathrm{~h}$ post TdR treatment and show a marked accumulation in Chk1-depleted cells. (b) Percentages of cells presenting low $(<10)$ or high $(>10)$ levels of foci. The error bars represent the S.D. of at least three independent experiments. More than 300 cells were counted for each condition in every experiment. (c) Formation of RPA34 and PCNA foci in control or Chk1 siRNA-treated cells exposed or not exposed to $2 \mathrm{mM}$ thymidine for $24 \mathrm{~h}$. Colocalization of RPA (red) and PCNA (green) appears as yellow. (d) Formation of Rad51 foci in HCT116 cells treated with control or Chk1 siRNAs following treatment with $2 \mathrm{mM}$ thymidine for $24 \mathrm{~h}$. Rad51 foci + cells were those that contained $>10$ foci. (e) Annexin V binding of Chk1-depleted HCT116 cells following $2 \mathrm{mM}$ TdR treatment for 24 or $48 \mathrm{~h}$. The percentage of Chk1depleted HCT116 cells presenting $>10$ RPA foci from panel (c) is also presented (dotted line)

fixed and stained for RPA foci. Interestingly, the hyperphosphorylation of RPA34 found in Chk1-depleted HCT116 cells treated with thymidine was not evident in cells depleted of
Cdc45 or Chk1 and Cdc45 (Figure 5c). Likewise, Cdc45 depletion alone had no significant effect on the fraction of cells showing RPA foci, relative to cells treated with the control 


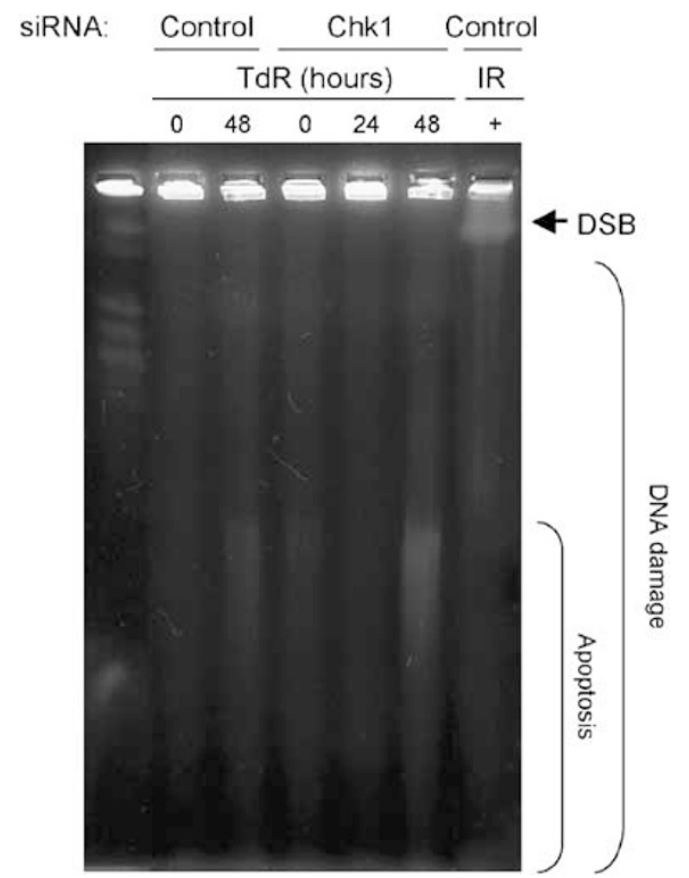

Figure 4 DSBs are not detectable in Chk1-depleted cells following replication fork stress. DSBs were visualized by pulsed-field gel electrophoresis of DNA obtained by gentle lysis of HCT116 cells in agarose plugs after the transfection with the indicated siRNAs, followed by the treatment or not with $2 \mathrm{mM}$ thymidine (TdR) for 24 or $48 \mathrm{~h}$. Agarose plugs treated with $10 \mathrm{~Gy}$ of IR were loaded as positive controls for DSBs. Regions of the gel showing DSBs, other DNA damage, and DNA fragments generated by apoptosis are indicated

siRNA following thymidine treatment (Figure $5 d$ and e). In contrast, there was a strong increase in the fraction of Chk1depleted cells accumulating RPA foci $(>10)$. When cells were depleted of both Cdc45 and Chk1, there was a substantial suppression of cells accumulating RPA foci and the pattern of foci was similar to that seen in cells treated with the control siRNA. Suppression of RPA foci formation was also found in SW480 cells depleted of Cdc45 and Chk1 following thymidine treatment (Figure 5f). Furthermore, this effect was not limited to cells treated with thymidine as hydroxyurea (HU) treated cells responded similarly (Figure $5 d$ and e). Thus, Chk1depleted cells show a marked accumulation of chromatinbound hyperphosphorylated RPA34 and RPA foci following treatment with agents that disrupt DNA replication, and this accumulation is Cdc45 dependent.

The induction of apoptosis in Chk1-depleted cells
treated with replication inhibitors is Cdc45 dependent. Given that Cdc45 depletion protected cells from the accumulation of RPA-ssDNA complex, we next determined whether it protected cells from apoptosis following treatment with replication inhibitors, by analyzing cell-cycle distribution of cultures treated with the Cdc45 siRNA. As we found earlier with unstressed cells, Cdc45 depletion also slowed the progression of stressed cells through S-phase. Cdc45-depleted HCT116 cells treated with replication inhibitors showed an increase in the fraction of cells in early S-phase and a decrease in the fraction in G2 relative to those treated with the control siRNA (Figure 6a). However, the fractions of cells with a subG1 DNA content were similar in cultures treated with the Cdc45 or control siRNAs. In contrast, Chk1-depleted cells treated with thymidine or $\mathrm{HU}$ showed a marked increase in the fraction with a subG1 DNA content. When HCT116 cells were depleted of both Cdc45 and Chk1, the level of cells with a subG1 DNA content was substantially reduced and was only slightly higher than that found in cells treated with control or Cdc45 siRNAs alone. Similar results were obtained with SW480 cells (Figure 6b). Furthermore measurements of Annexin $\mathrm{V}+$ cells in cultures treated with thymidine or $\mathrm{HU}$ revealed a similar pattern of apoptotic cells (Figure 6c). Cells depleted of Chk1 showed an increase in the fraction of Annexin $\mathrm{V}+$ cells relative to controls while the level of Annexin V + cells in cultures depleted of both Cdc45 and Chk1 was reduced to that found in cultures treated with the control siRNA. Finally we measured colony formation by HCT116 cells depleted of Cdc45 and/or Chk1 in thymidine (Figure 6d). siRNA-mediated depletion of Cdc45 alone had no significant effect on colony formation relative to cells treated with the control siRNA, whereas Chk1 depletion caused a marked reduction in colony formation. Depletion of both proteins restored colony formation in thymidine to that measured in the control cultures. Thus, Cdc45 depletion protects cells from the apoptotic effects of Chk1 depletion during replication stress.

p21 protects Chk1-depleted cells from the accumulation of RPA foci and apoptosis following replication stress. p21 exerts a negative effect on replication initiation through its role as a cyclin-dependent kinase inhibitor. ${ }^{27}$ In Chk1-depleted cells treated with thymidine, p21 is induced in a p53-independent manner. ${ }^{16}$ As shown previously, a significantly higher fraction of p21-deficient cells depleted of Chk1 have a subG1 DNA content relative to p21-proficient cells (Figure $7 a$ and $b$ ), suggesting that $p 21$ may provide some protection from apoptosis in the absence of Chk1 by reducing origin firing. To determine whether loss of p21 correspondingly increased the fraction of cells accumulating RPA foci, these were measured in HCT116 p21 $+/+$ and p21-/- cells depleted of Chk1 and treated with thymidine. Although the fraction of cells showing RPA foci in the presence of Chk1 was not affected by p21 status, a significantly higher proportion of p21-deficient cells accumulated RPA foci following Chk1 depletion relative to p21-proficient cells (Figure 7c). Thus, a negative effector of origin firing suppresses both the accumulation of RPA foci and the induction of apoptosis in Chk1-depleted cells treated with thymidine.

\section{Discussion}

Chk1 triggers a complex network of responses to the disruption of DNA replication. Here we investigated the Chk1-mediated response that protects cells from apoptosis. The work presented here argues that its role in controlling inappropriate firing of replication origins is particularly critical to prevent cell death (for model, see Figure 8). We show that RPA is recruited to chromatin and RPA foci form in cells 


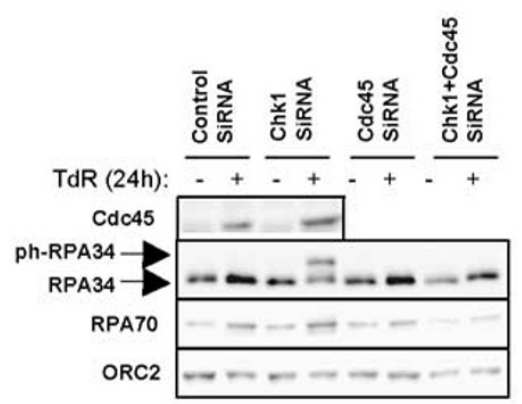

b

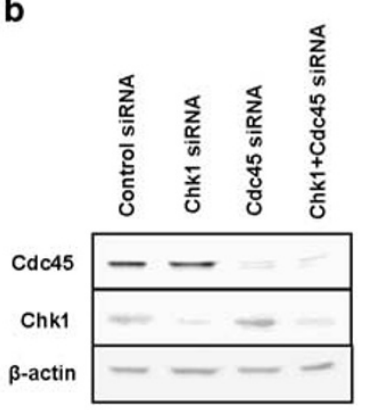

C

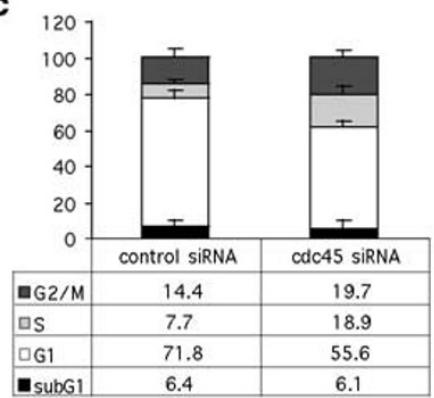

d

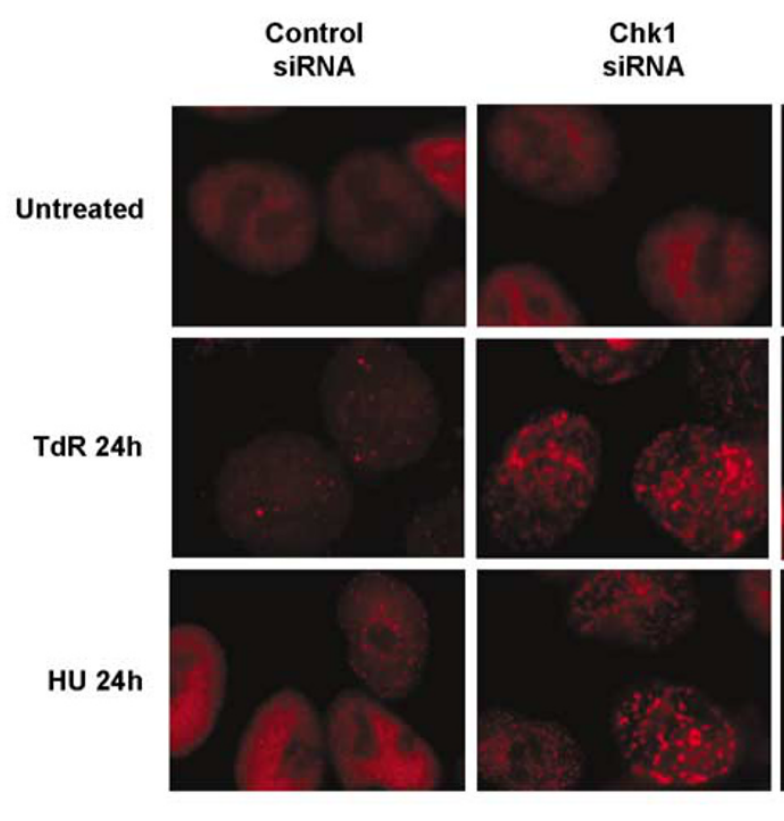

e

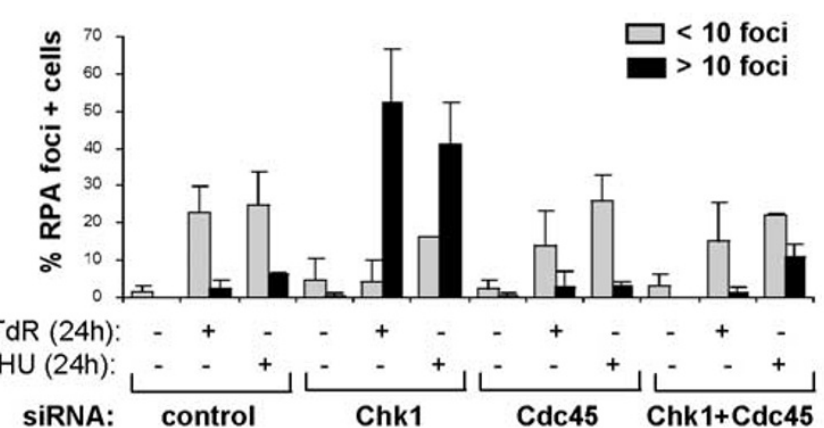

f

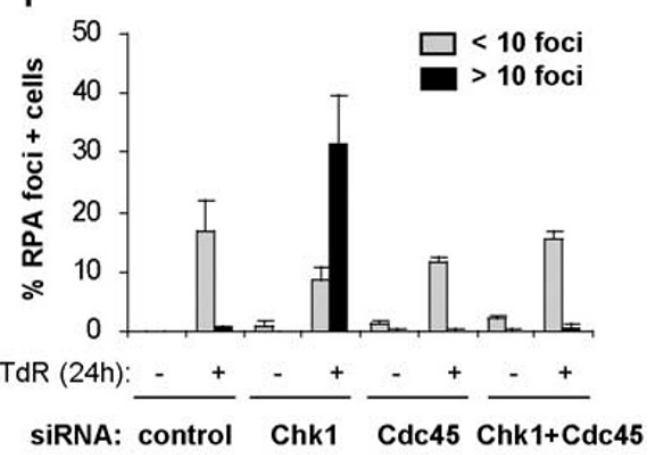

Figure 5 Cdc45 knockdown suppresses the accumulation of RPA foci in Chk1-depleted cells. (a) Protein levels of Cdc45, RPA34, RPA70, and ORC2 (as loading control) present in the chromatin-bound fractions of HCT116 cells transfected with the indicated siRNAs, and treated or not treated with $2 \mathrm{mM}$ thymidine (TdR) for $24 \mathrm{~h}$. The hyperphosphorylated form of RPA34 is indicated. (b) Chk1 and Cdc45 levels $24 \mathrm{~h}$ after the transfection of indicated siRNAs in HCT116 cells. (c) Percentage of cells in each phase of the cell cycle $48 \mathrm{~h}$ after the transfection of the indicated siRNAs. (d, e) Immunostaining of RPA34 after the treatment of HCT116 cells transfected with the indicated siRNAs with $2 \mathrm{mM}$ thymidine (TdR) or $2 \mathrm{mM} \mathrm{HU}$ for $24 \mathrm{~h}$. (d) Representative images show RPA staining following Cdc45 and/or Chk1 depletion. The frequency of Chk1depleted cells accumulating RPA foci is strongly suppressed in cells that are also depleted of Cdc45. (e) Percentages of cells presenting low $(<10)$ or high $(>10)$ levels of foci. The error bars represent the S.D. of at least three independent experiments. More than 300 cells were counted for each condition in every experiment. (f) Percentages of SW480 cells presenting low $(<10)$ or high $(>10)$ level of RPA foci

treated with replication inhibitors, consistent with the formation of RPA-ssDNA complex that has been shown to occur as a result of uncoupling of the polymerase and helicase complexes in Xenopus egg extracts. ${ }^{7}$ In Chk1-depleted cells, agents that disrupt replication cause a dramatic increase in the number of these RPA foci and RPA34 hyperphosphorylation. We propose that the inappropriate firing of replication origins that occurs in Chk1-depleted cells ${ }^{6,10,12}$ increases the 

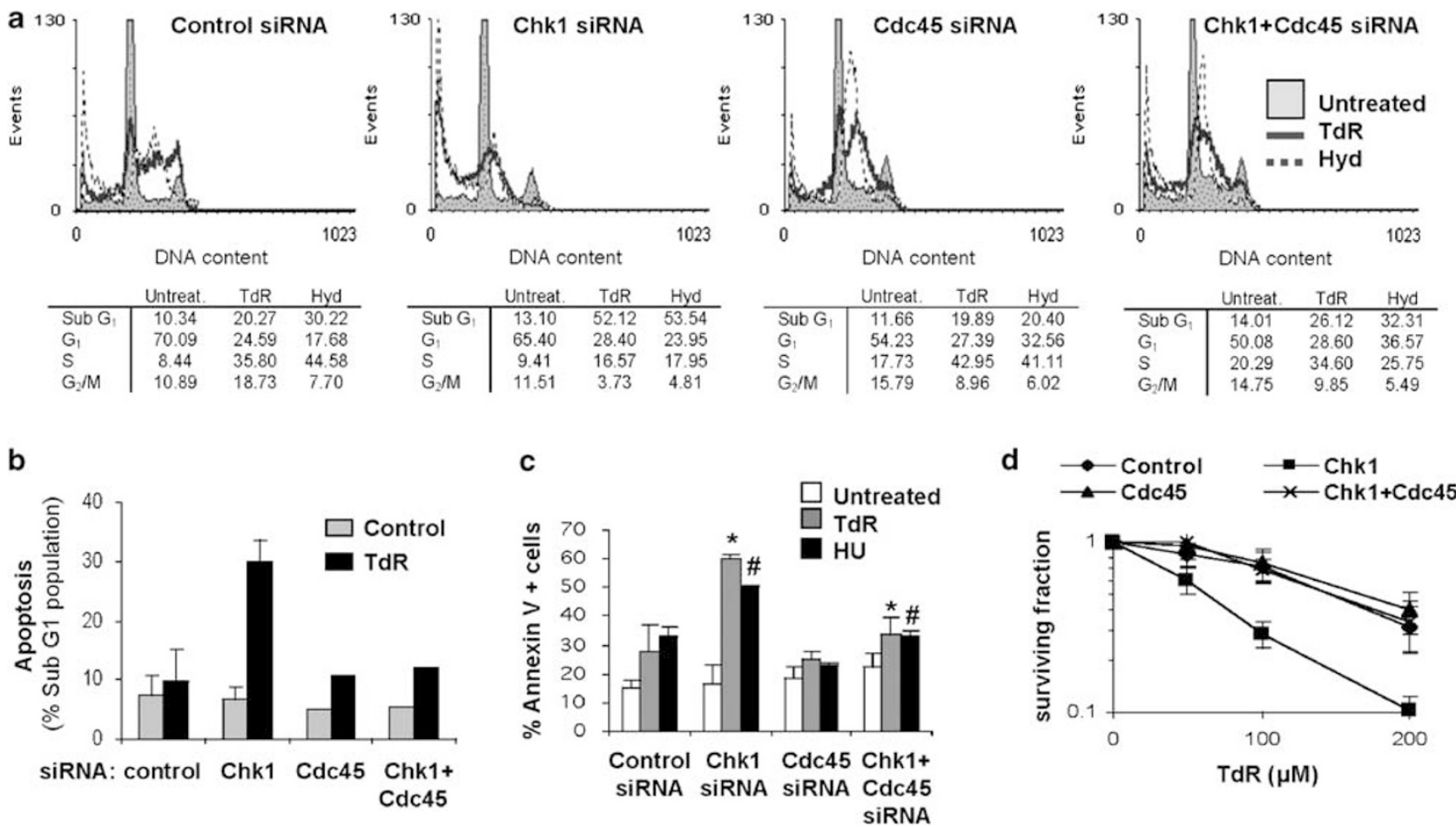

Figure 6 Cdc45 knockdown prevents the induction of apoptosis in Chk1-depleted cells. (a) HCT116 or (b) SW480 cells transfected with the indicated siRNAs for $24 \mathrm{~h}$ were treated with $2 \mathrm{mM}$ thymidine (TdR) or $2 \mathrm{mM} \mathrm{HU}$ for $48 \mathrm{~h}$ and analyzed for cell-cycle distribution. (c) HCT116 cells treated as above were analyzed for Annexin V binding $\left({ }^{*} P<0.01 ;{ }^{\#} P>0.005\right)$. (d) Colony formation of HCT116 cells depleted of Chk1 and/or Cdc45 in TdR. HCT116 cultures were transfected with the indicated siRNAs as in panel (a). Twenty-four hours post transfection, the cultures were trypsinized and plated in the indicated concentrations of TdR. Chk1 and/or Cdc45 depletion was monitored by western blotting using parallel cultures treated with the siRNAs (data not shown)

number of forks where ssDNA-RPA complex accumulates as an early event following replication fork stress. In support of this proposal, depletion of the helicase cofactor Cdc45 suppresses the induction of RPA foci and apoptosis. Whereas this protein is required for both the initiation and elongation steps of DNA synthesis, disruption of either would retard the formation of inappropriate replication fork intermediates in Chk1-depleted cells. In contrast, loss of p21, a negative effector of replication origin firing that is normally induced in Chk1-depleted cells, ${ }^{16}$ leads to a further increase in the fraction of cells accumulating RPA foci and undergoing apoptosis.

An alternative explanation of these observations is that the apoptotic response may be the result of DNA damage accumulating in Chk1-depleted cells treated with replication inhibitors and/or aberrant repair. However, several lines of evidence argue against this explanation. First, the primary agent we used in these experiments (thymidine) did not generate detectable DSBs that might form as a result of the collapse of stressed forks in the presence or absence of Chk1. Second, the status of the two repair pathways that deal with replication fork stress induced by thymidine did not determine the apoptotic response. DNA replication errors generated by thymidine ${ }^{28}$ are repaired by MMR, while HR is required for efficient S-phase progression by promoting restart of stalled replication forks. ${ }^{20}$ The apoptotic response obtained in Chk1depleted cells treated with thymidine was similar in both MMRdeficient (HCT116) and MMR-proficient (SW480) cells. We further showed that there was no increase in the level of apoptosis in response to replication fork stress in cells deficient in HR. Finally the apoptotic response was dependent upon $\mathrm{Cdc} 45$. Although it is not yet clear whether Cdc45 plays any role in repair pathways in mammalian cells, in yeast Cdc45 is absolutely required for genomic replication but not for HR-mediated DSB gene conversion. ${ }^{25}$ Experiments with Xenopus egg extract systems provide strong evidence that the uncoupling of the Cdc45-MCM helicase from the replication complex generates RPA-ssDNA complex when polymerase activity is arrested. ${ }^{7}$ The Cdc45 dependence of RPA foci accumulation in Chk1-depleted cells treated with replication inhibitors provides evidence that this mechanism functions in vivo. Furthermore, our previous observation that loss of the cyclin-dependent kinase inhibitor p21 affects death at the G1-S border, ${ }^{16}$ argues that its role in the control of replication initiation is crucial for the control of apoptosis under these conditions. Taken together, these observations support our argument that the role of Chk1 in the control of aberrant replication initiation is crucial to prevent apoptosis.

The signal that finally triggers the apoptotic response is not revealed by these experiments. Chk1 depletion alone is not sufficient to trigger apoptosis in our cells. It is only after addition of inhibitors of replication elongation that apoptosis occurs. RPA foci or hyperphosphorylated RPA34 that accumulate under these conditions are attractive candidates for such a signalling function. RPA has been shown to interact with a number of proteins involved in the DNA-damage 


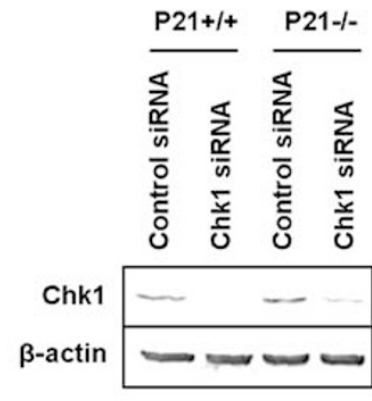

b

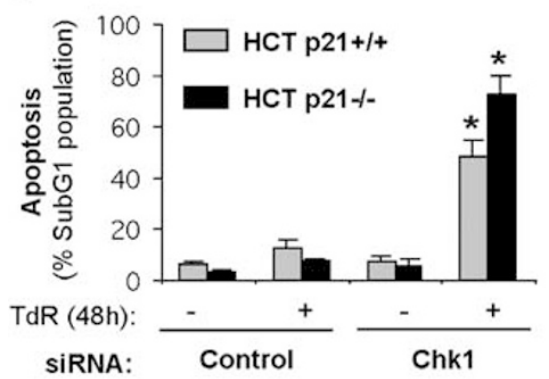

C

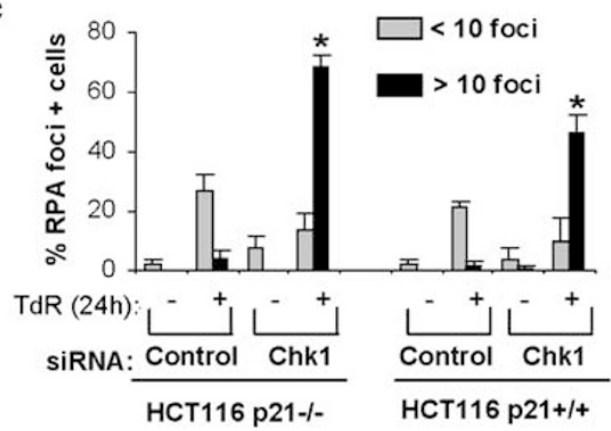

Figure 7 p21 protects Chk1-depleted cells from the accumulation of RPA foci and apoptosis following replication stress. HCT116 p21 + / + or HCT116 p21-/cells were transfected with the indicated siRNAs for $24 \mathrm{~h}$ and analyzed for (a) Chk1 and $\beta$-actin protein levels. (b) Percentage of cells showing subG1 DNA content after a 48-h treatment with $\left.2 \mathrm{mM} \mathrm{TdR} \mathrm{(}{ }^{*} P<0.01\right)$. (c) Immunostaining of RPA34. The percentages of cells presenting low $(<10)$ or high $(>10)$ levels of foci are shown $\left({ }^{*} P<0.02\right)$. The error bars represent the S.D. of at least two independent experiments. More than 300 cells were counted for each condition in every experiment

response, such as ATR-ATRIP or the Rad9-Rad1-Hus1 complex. ${ }^{8,9,29}$ However, attempts to test this hypothesis by siRNA-mediated depletion of RPA34 or RPA70 have not been successful. Consistent with another report, ${ }^{30}$ RPA34 depletion induced a G2 arrest only at later times after treatment (data not presented). Since the effects of Chk1 depletion at replication forks occur earlier, the usefulness of this approach appears limited.

The targeted knockout of Chk1 in mice leads to early embryonic lethality. ${ }^{31}$ In mouse ES cells, disruption of Chk1 induces spontaneous apoptosis that is the result of mitotic catastrophe caused by premature activation of Cdc2cyclinB. ${ }^{32}$ We found only a very low level of mitotic catastrophe in our Chk1-depleted cells either in the presence or absence of replication inhibitors. ${ }^{16}$ Importantly, it has been reported that mouse ES cells have a defective p21 response to DNA damage ${ }^{33}$ that, in combination with Chk1 deficiency, could enhance the apoptotic response of these cells. Thus, the cellular response to Chk1 depletion appears to depend upon the stringency of the depletion and the integrity of responses to genomic stress.

Recent studies have indicated that some tumors show evidence of a chronic activation of DNA-damage responses that might be the result of replication stress driven by activated oncogenes. ${ }^{34,35}$ Tumor microenvironment may also contribute to replication fork stress. Hypoxia induces alterations in dNTP pool levels that could lead to replication stress ${ }^{36}$ and trigger the ATR-Chk1-signalling pathway. ${ }^{37}$ The mechanism presented here suggests that activated Chk1 would play a critical role in protecting cells from apoptosis at such points in tumor development. Therefore, treatment strategies targeting Chk1 in combination with replication inhibitors might prove particularly effective against tumors showing Chk1 activation.

\section{Materials and Methods}

Cell lines and cultures. The HCT116 human colon cancer cell line was obtained from ATCC. The p21 derivative of this line was generously provided by $\mathrm{Dr}$ Bert Vogelstein (Johns Hopkins University, Baltimore, MD). The isolation of SW480/ SN3 was described previously. ${ }^{38}$ Cells were maintained in DMEM supplemented with $10 \%$ fetal bovine serum (FBS). For experiments using thymidine (Sigma), dialyzed FBS was used to remove deoxynucleosides that might alter the cellular response to this agent.

siRNA transfection. Chk1 siRNA was designed to correspond to the Chk1 DNA sequence (J Blackburn and C Smythe, unpublished data). Rad51 (M-00353003), XRCC3 (M-012067-00), and Cdc45 (M-003232-02) siRNAs were from Dharmacon (Lafayette, CO) and the control siRNA (OR-0030-NEG) was from Eurogenetec. siRNA duplexes were transfected into cells using Lipofectamine 2000 (Invitrogen) according to the manufacturer's instructions. In experiments where the effect of a single siRNA was compared with the effect of two siRNAs, control siRNA was used to keep the amount of siRNA constant. Twenty-four hours after transfection, cells were washed with phosphate-buffered saline (PBS) before treatment for 24 or $48 \mathrm{~h}$. Cells were then collected and analyzed.

Colony-forming assay. Cultures were transfected with different siRNAs for $24 \mathrm{~h}$ and then trypsinized and plated in triplicate at low density $(1000 \mathrm{celll} / 100-\mathrm{mm}$ dish) in media containing the indicated levels of thymidine. Depletion of relevant proteins was confirmed by western blotting using extracts prepared from paralle cultures treated with the siRNAs. Cells were grown 10-15 days before staining with $0.4 \%$ methylene blue (Sigma) $/ 50 \%$ methanol (Fisher Scientific). Colonies of $>50$ cells were scored. The surviving fraction was determined by dividing the average number of colonies for each treatment by the average number of colonies in the control.

Cell-cycle analysis. Cell-cycle analysis of floating and adherent cells was carried out as described previously. ${ }^{16}$

Detection of apoptosis. Apoptotic cells were assessed by flow cytometry using FITC-Annexin V and propidium iodide or TUNEL staining according to the manufacturer's instructions (BD Biosciences).

Detection of DSBs by pulsed-field gel electrophoresis. HCT116 cells were treated with $2 \mathrm{mM}$ thymidine for $48 \mathrm{~h}$. Following treatments, $1 \times 10^{6}$ cells were embedded into agarose inserts. For IR, $1 \times 10^{6}$ cells were melted into an agarose insert and then treated with $10 \mathrm{~Gy}$ of IR. The agarose inserts were incubated in $0.5 \mathrm{M}$ EDTA, $1 \% \mathrm{~N}$-laurylsarcosyl, and proteinase $\mathrm{K}(1 \mathrm{mg} / \mathrm{ml})$ for $48 \mathrm{~h}$ and then washed four times in TE buffer, before loading onto a $1 \%$ agarose gel. Pulsed-field gel electrophoresis ( $120^{\circ}$ angle, $60-240$ s switch time, $4 \mathrm{~V} / \mathrm{cm}$; BioRad) was then carried out. The gel was subsequently stained with ethidium bromide and analyzed using Image Gauge software. 
a

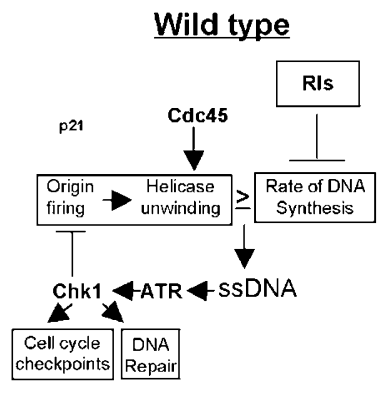

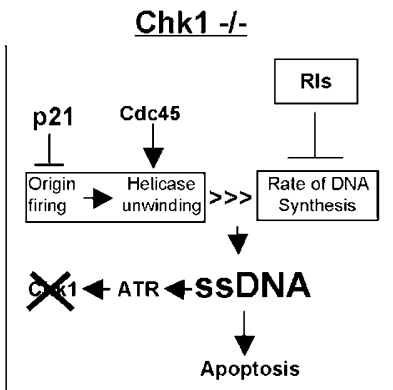

Chk1 -/-; Cdc45 -/-

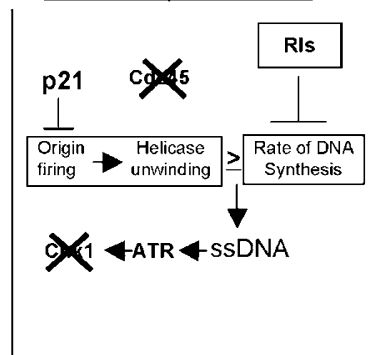

Figure 8 Model for the role of the RPA-ssDNA complex as a apoptotic signal. Inhibition of replication polymerase in wild-type cells by replication inhibitors (RIs) causes a decrease in the rate of DNA synthesis and generates RPA-coated ssDNA regions as a result of the continued function of the helicase complex (a). This activates the ATRChk1-signalling pathway, which in turn inhibits inappropriate origin firing and prevents the further accumulation of ssDNA regions (as detected by formation of RPA foci). This allows time for repair and the restart of DNA synthesis. Chk1-depleted cells cannot prevent inappropriate origin firing and the uncoupling of the helicase and polymerase activities causes accumulation of ssDNA (b). We propose that the accumulation of ssDNA provides a signal that is amplified in the absence of Chk1 to trigger apoptosis. After Chk1 depletion, p21 is upregulated and partially prevents this apoptotic signal. When the helicase cofactor Cdc45 is knocked down in Chk1-depleted cells, replicative helicase activity is low, preventing the accumulation of SSDNA and cell death (c)

Recombination assay. The SCneo recombination reporter-containing cell line SW480/SN3 was transfected with the indicated siRNA as described above. Twenty-four hours later they were transfected either with and empty vector or a pCMV3nls-I-Scel expression vector for another $24 \mathrm{~h}$ and analyzed for frequency of neo ${ }^{+}$recombinants as described previously. ${ }^{38}$

Cell extraction and western blotting. Whole-cell extracts were prepared as described previously. ${ }^{21}$ Chromatin-bound proteins were isolated as described by $\mathrm{Li}$ and Stern. ${ }^{19} \mathrm{~A}$ total of $3 \times 10^{6}$ cells were washed with PBS, resuspended in $200 \mu$ l of solution A $\left(10 \mathrm{mM} \mathrm{HEPES}\right.$ at $\mathrm{pH} 7.9,10 \mathrm{mM} \mathrm{KCl}, 1.5 \mathrm{mM} \mathrm{MgCl}_{2}, 0.34 \mathrm{M}$ sucrose, $10 \%$ glycerol, $1 \mathrm{mM}$ dithiothreitol, $10 \mathrm{mM} \mathrm{NaF}, 1 \mathrm{mM} \mathrm{Na}_{2} \mathrm{VO}_{3}$, and protease inhibitors) containing $0.1 \%$ of Triton $\mathrm{X}-100$, and incubated on ice for $5 \mathrm{~min}$. Cytoplasmic proteins (supernantant $1, \mathrm{~S} 1$ ) were separated from nuclei by low-speed centrifugation $\left(1300 \times g\right.$ for $4 \mathrm{~min}$ at $\left.4^{\circ} \mathrm{C}\right)$. Isolated nuclei were washed once with solution $A$ and then lysed in $200 \mu$ l of solution B ( 3 mM EDTA, 0.2 mM EGTA, $1 \mathrm{mM}$ dithiothreitol, and protease inhibitors) on ice for $10 \mathrm{~min}$. Soluble nuclear proteins (S2) were separated from insoluble chromatin by centrifugation $(1700 \times \mathrm{g}$ for $4 \mathrm{~min}$ at $4{ }^{\circ} \mathrm{C}$ ). Isolated chromatin was washed once with solution $B$ and centrifuged at $10000 \times g$ for $1 \mathrm{~min}$. The final chromatin (pellet, P) was resuspended in $200 \mu \mathrm{l}$ of Laemmli buffer and boiled for $5 \mathrm{~min}$. Proteins were separated by SDS-PAGE and blotted onto nitrocellulose (Schleicher \& Schuell). Proteins were detected using the enhanced chemiluminiscence detection system (ECL, Amersham) according to the manufacturer's recommendations, using anti-Chk1 (no. 2345) from Cell Signaling; anti-Rad51 (sc-8349), anti-XRCC3 (sc-5904), anti-Cdc45 (sc-20685), and antiRPA70 (sc-14696) from Santa Cruz Technology; anti-RPA34 (NA19L) from Calbiochem; anti-ORC2 (no. 559266) and anti-GRB2 (no. 610111) from BD Pharmingen; or anti- $\beta$-actin (A-5060) from Sigma.

Immunofluorescence staining. For RPA or Rad51 foci staining, cells were cultured on glass coverslips, treated as indicated, fixed with $4 \%$ formaldehyde for $20 \mathrm{~min}$ at room temperature (RT), and permeabilized in PBS containing $0.5 \%$ Nonidet P-40 for 5 min at RT. Cells were then blocked with PBS containing $1 \%$ BSA and $0.05 \%$ Tween 20 (PBT) for $5 \mathrm{~min}$ at RT and incubated with $2 \mu \mathrm{g} / \mathrm{ml}$ anti-RPA34 (NA19L; Calbiochem) or 1:100 diluted anti Rad51 (sc-8349) for $1 \mathrm{~h}$ and 1:100 diluted Texas Red-conjugated donkey anti-mouse IgG (no. 715-075-151; Jackson ImmunoResearch Laboratories) or FITC-conjugated anti-rabbit IgG (no. 711-095152; Jackson ImmunoResearch Laboratories) for $1 \mathrm{~h}$ in the dark. Antibody dilutions and washes after incubations were performed in PBT. For the simultaneous analysis of RPA and PCNA foci, cells were first fixed, permeabilized, and stained for RPA as above. Cells were then fixed again with ice-cold methanol for $5 \mathrm{~min}$, washed twice with PBT, and incubated with 1:100 diluted FITC conjugated-anti PCNA antibody (sc-56FITC; Santa Cruz Technology) for $1 \mathrm{~h}$ in the dark. Coverslips were finally mounted in Vectashield mounting medium with DAPI (H-1500; Vector Laboratories Inc.). The resulting fluorescence was analyzed using a Nikon Eclipse T200 microscopy equipped with a Hamamatsu Orca ER camera and the Volocity
3.6.1 (Improvision) software. Rad51 foci were assayed as described by Sorensen et $a .^{13}$

Acknowledgements. We are grateful to Helen Bryant, Cyril Sanders, and Kirsteen Maclean for their comments concerning the paper. This work was supported by a programme grant from Yorkshire Cancer Research to MM.

1. Zhou B-BS, Elledge SJ. The DNA damage response: putting checkpoints in perspective. Nature 2000; 408: 433-439.

2. Kastan MB, Bartek J. Cell-cycle checkpoints and cancer. Nature 2004; 432: 316-323.

3. Ward IM, Minn K, Chen J. UV-induced ataxia-telangiectasia-mutated and Rad3-related (ATR) activation requires replication stress. J Biol Chem 2004; 279: 9677-9680.

4. Cliby WA, Roberts CJ, Cimprich KA, Stringer CM, Lamb JR, Schreiber SL et al. Overexpression of a kinase-inactive ATR protein causes sensitivity to DNA-damaging agents and defects in cell cycle checkpoints. EMBO J 1998; 17: 159-169.

5. Brown EJ, Baltimore D. Essential and dispensable roles of ATR in cell cycle arrest and genome maintenance. Genes Dev 2003; 17: 615-628.

6. Zachos G, Rainey MD, Gillespie DA. Chk1-deficient tumour cells are viable but exhibit multiple checkpoint and survival defects. EMBO J 2003; 22: 713-723.

7. Byun TS, Pacek M, Yee MC, Walter JC, Cimprich KA. Functional uncoupling of MCM helicase and DNA polymerase activities activates the ATR-dependent checkpoint. Genes Dev 2005; 19: 1040-1052.

8. Zou L, Elledge SJ. Sensing DNA damage through ATRIP recognition of RPA-ssDNA complexes. Science 2003; 300: 1542-1548.

9. Ball HL, Myers JS, Cortez D. ATRIP binding to replication protein A-single-stranded DNA promotes ATR-ATRIP localization but is dispensible for Chk1 phosphorylation. Mol Biol Cell 2005; 16: 2372-2381.

10. Feijoo C, Hall-Jackson C, Wu R, Jenkins D, Leitch J, Gilbert DM et al. Activation of mammalian Chk1 during DNA replication arrest: a role for Chk1 in the intra-S phase checkpoint monitoring replication origin firing. J Cell Biol 2001; 154: 913-923.

11. Nghiem P, Park PK, Kim Y, Vaziri C, Schreiber SL. ATR inhibition selectively sensitizes G1 checkpoint-deficient cells to lethal premature chromatin condensation. Proc Natl Acad Sci USA 2001; 98: 9092-9097.

12. Maya-Mendoza A, Petermann E, Gillespie DA, Caldecott KW, Jackson DA. Chk1 regulates the density of active replication origins during the vertebrate S phase. EMBO J 2007; 26: 2719-2731.

13. Sorensen CS, Hansen LT, Dziegielewski J, Syljuasen RG, Lundin C, Bartek J et al. The cell-cycle checkpoint kinase Chk1 is required for mammalian homologous recombination repair. Nat Cell Biol 2005; 7: 195-201.

14. Cho SH, Toouli CD, Fujii GH, Crain C, Parry D. Chk1 is essential for tumor cell viability following activation of the replication checkpoint. Cell Cycle 2005; 4: 131-139.

15. Xiao Z, Xue J, Sowin TJ, Rosenberg SH, Zhang H. A novel mechanism of checkpoint abrogation conferred by Chk1 downregulation. Oncogene 2005; 24: 1403-1411.

16. Rodriguez R, Meuth M. Chk1 and p21 cooperate to prevent apoptosis during DNA replication fork stress. Mol Biol Cell 2006; 17: 402-412.

17. Johnson RD, Liu N, Jasin M. Mammalian XRCC2 promotes the repair of double-strand breaks by homologous recombination. Nature 1999; 401: 397-399. 
18. Hinz JM, Helleday T, Meuth M. Reduced apoptotic response to camptothecin in $\mathrm{CHO}$ cells deficient in XRCC3. Carcinogenesis 2003; 24: 249-253.

19. Li J, Stern DF. DNA damage regulates Chk2 association with chromatin. J Biol Chem 2005 280: 37948-37956.

20. Lundin C, Erixon K, Arnaudeau C, Schultz N, Jenssen D, Meuth M et al. Different roles for nonhomologous end joining and homologous recombination following replication arrest in mammalian cells. Mol Cell Biol 2002; 22: 5869-5878.

21. Bolderson E, Scorah J, Helleday T, Smythe C, Meuth M. ATM is required for the cellular response to thymidine induced replication fork stress. Hum Mol Genet 2004; 13 2937-2945.

22. Zou L, Mitchell J, Stillman B. CDC45, a novel yeast gene that functions with the origin recognition complex and Mcm proteins in initiation of DNA replication. Mol Cell Biol 1997 17: 553-563.

23. Pacek M, Walter JC. A requirement for MCM7 and Cdc 45 in chromosome unwinding during eukaryotic DNA replication. EMBO J 2004; 23: 3667-3676.

24. Pacek M, Tutter AV, Kubota $Y$, Takisawa $H$, Walter JC. Localization of the MCM2-7, $\mathrm{Cdc45}$, and GINS to the site of DNA unwinding during eukaryotic DNA replication. Mol Cell 2006; 21: 581-587.

25. Wang X, Ira G, Tercero JA, Holmes AM, Diffley JF, Haber JE. Role of DNA replication proteins in double-strand break-induced recombination in Saccharomyces cerevisiae. $\mathrm{Mol}$ Cell Biol 2004; 24: 6891-6899.

26. Syljuasen RG, Sorensen CS, Hansen LT, Fugger K, Lundin C, Johansson F et al. Inhibition of human Chk1 causes increased initiation of DNA replication, phosphorylation of ATR targets, and DNA breakage. Mol Cell Biol 2005; 25: 3553-3562.

27. El-Deiry WS, Tokino T, Velculescu VE, Levy DB, Parsons R, Trent JM et al. WAF1, publisher: a potential mediator of p53 tumor suppression. Cell 1993; 75: 817-825.

28. Meuth $M$. The molecular basis of mutations induced by deoxyribonucleotide triphosphate pool imbalances in mammalian cells. Exp Cell Res 1989; 181: 305-316.
29. Zou Y, Liu Y, Wu X, Shell SM. Functions of human replication protein A (RPA): from DNA replication to DNA damage and stress responses. J Cell Physiol 2006; 208: 267-273.

30. Dodson GE, Shi Y, Tibbetts RS. DNA replication defects, spontaneous DNA damage, and ATM-dependent checkpoint activation in replication protein A deficient cells. J Biol Chem 2004; 279: 34010-34014

31. Takai $\mathrm{H}$, Tominaga $\mathrm{K}$, Motoyama $\mathrm{N}$, Minamishima $\mathrm{YA}$, Nagahama $\mathrm{H}$, Tsukiyama $\mathrm{T}$ et al. Aberrant cell cycle checkpoint function and early embryonic death in Chk1-/- mice. Genes Dev 2000; 14: 1439-1447.

32. Niida H, Tsuge S, Katsuno Y, Konishi A, Takeda N, Nakanishi M. Depletion of Chk1 leads to premature activation of Cdc2-cyclinB and mitotic catastrophe. J Biol Chem 2005; 280 39246-39252.

33. Hong Y, Stambrook PJ. Restoration of an absent G1 arrest and protection from apoptosis in embryonic stem cells after ionizing radiation. Proc Natl Acad Sci USA 2004; 101 14443-14448.

34. Bartkova J, Horejsi Z, Koed K, Kramer A, Tort F, Zieger K et al. DNA damage response as a candidate anti-cancer barrier in early human tumorigenesis. Nature 2005; 434: 864-870.

35. Gorgoulis VG, Vassiliou LV, Karakaidos P, Zacharatos P, Kotsinas A, Liloglou T et al. Activation of the DNA damage checkpoint and genomic instability in human precancerous lesions. Nature 2005; 434: 907-913.

36. Chimploy K, Tassotto ML, Mathews CK. Ribonucleotide reductase, a possible agent in deoxyribonucleotide pool asymmetries induced by hypoxia. J Biol Chem 2000; 275 39267-39271.

37. Hammond EM, Dorie MJ, Giaccia AJ. ATR/ATM targets are phosphorylated by ATR in response to hypoxia and ATM in response to reoxygenation. $J$ Biol Chem 2003; 278 12207-12213.

38. Mohindra A, Hays LE, Phillips EN, Preston BD, Helleday T, Meuth M. Defects in homologous recombination repair in mismatch-repair-deficient tumour cell lines. Hum Mol Genet 2002; 11: 2189-2200.

Supplementary Information accompanies the paper on Cell Death and Differentiation website (http://www.nature.com/cdd) 